\title{
Antagonism of the Gerbil's Sucrose Taste Response by p-Nitrophenyl $\alpha$-D-Glucopyranoside and Chloramphenicol
}

\author{
V. Vlahopoulos and W. Jakinovich, Jr. \\ Department of Biological Sciences, Lehman College, and the Graduate School, City University of New York, \\ Bronx, New York 10468
}

We have discovered that the gerbil's chorda tympani nerve response to sucrose is suppressed by $p$-nitrophenyl $\alpha$-D-glucopyranoside (PNP-Glu) and chloramphenicol (CAP). Mixture experiments of PNP-Glu and CAP with sodium chloride, potassium chloride, hydrochloric acid, and sucrose gave rise to the following observations: (1) Neither PNP-Glu nor CAP alone stimulates the gerbil's taste nerve; (2) while the sucrose response is suppressed by these inhibitors, taste responses produced by sodium chloride, potassium chloride, and hydrochloric acid are unaffected by the presence of PNP-Glu or CAP; (3) the antagonisms of PNP-Glu and CAP were surmounted by a high concentration of sucrose; (4) CAP is a more potent antagonist $\left(\mathrm{IC}_{50}=\right.$ $0.0013 \mathrm{M}$ ) than PNP-Glu $\left(\mathrm{IC}_{50}=0.022 \mathrm{M}\right)$, and both are more potent than methyl 4,6-dichloro-4,6-dideoxy- $\alpha$-D-galactopyranoside $\left(\mathrm{IC}_{\mathrm{s0}}=0.048 \mathrm{M}\right)$; and (5) sucrose antagonism occurs only when PNP-Glu and CAP are mixed with sucrose. It is shortlived and ceases when the mixtures are rinsed from the gerbil's tongue.

Structure-activity studies provided the following information: (1) The alpha anomer of PNP-Glu is a more potent inhibitor than its beta anomer; (2) among the PNP-Glu derivatives tested (p-aminophenyl, $p$-nitrophenyl, and phenyl) only $p$-nitrophenyl inhibited; (3) among the nitrophenyl galactosides, the para derivative was more potent than the ortho or meta; and (4) $p$-nitrophenyl $\alpha$-D-mannopyranoside and $p$-nitrophenyl $\alpha$-D-galactoside are slightly more potent than PNP-Glu.

On the basis of concentration experiments, we believe that the inhibitory mechanisms of PNP-Glu and CAP are different, which suggests the existence of at least 2 sucrose receptor sites.

Recently (Jakinovich, 1983) we discovered an antagonist of the gerbil's sucrose taste response, methyl 4,6-dichloro-4,6-dideoxy- $\alpha$-D-galactopyranoside (MAD-diCl-Gal), a compound that resembles part of the sweetener molecule, chloro-sucrose $(4,6-$ dichloro-4,6-dideoxy- $\alpha$-D-galactopyranosyl- $1^{\prime}, 6^{\prime}$-dichloro- $1^{\prime}, 6^{\prime}$ dideoxy- $\beta$-D-fructofuranoside) (Hough and Phadnis, 1976). As a model compound to be used in further studies of sweet taste, MAD-diCl-Gal has some shortcomings. Therefore, we continued our search for better inhibitors amongst MAD-diCl-Gal derivatives, which led to the synthesis and testing of chlorinated methyl glycosides (Vlahopoulos and Jakinovich, 1986). However, none of these compounds proved to be more potent inhibitors than MAD-diCl-Gal.

Our next approach in this study was to test other chlorinated glycosides modified at the $\mathrm{C}-1$ position. Since $p$-nitrophenyl

\footnotetext{
Received Nov. 26, 1985; revised Feb. 24, 1986; accepted Mar. 18, 1986.

We thank D. Sugarman for manuscripl suggestions, A. Ward for typing, and P. Blochiaviak for doing the rotations. This research was supported by Grant ROINS 16022 from the NIH and PSC-CUNY.

Correspondence should be addressed to William Jakinovich, Jr., Department of Biological Sciences, Lehman College, CUNY, Bedford Park Blvd. West, Bronx, NY 10468

Copyright (C) 1986 Society for Neuroscience $0270-6474 / 86 / 092611-05 \$ 02.00 / 0$
}

$\alpha$-D-glucopyranoside (PNP-Glu) is also a sugar derivative and readily available, we decided to use it as starting material for the synthesis of a putative inhibitor, $p$-nitrophenyl 4,6-dichloro4,6-dideoxy- $\alpha$-D-galactopyranoside (PNP-diCl-Gal). As is the practice in our lab (Jakinovich, 1981-1983; Vlahopoulos and Jakinovich, 1986), we always test new sweetener derivatives on the gerbil's sucrose taste response. In this case, we found that the PNP-Glu, while nonstimulatory by itself, specifically suppressed the sucrose response when used in the mixture with sucrose. Surprisingly, it turned out to be a more potent inhibitor than MAD-diCl-Gal, and was less bitter.

We later tested other closely related compounds containing the $p$-nitrophenyl group. One of these, chloramphenicol (CAP), which, on the basis of our structure-activity predictions, should not have been a good inhibitor, actually turned out to be the most potent inhibitor of the 3 .

\section{Materials and Methods}

Materials

Animals. Mongolian gerbils (Meriones unguiculatus) were obtained from Tumblebrook Farm, West Brookfield, MA; they were less than 1 year old and weighed $50-70 \mathrm{gm}$.

Compounds. The compounds used in the experiments were purchased from Sigma Chemical Company, St. Louis, MO.

Taste solutions. All compounds were dissolved in deionized water and either used immediately, stored at $2^{\circ} \mathrm{C}$ for $7 \mathrm{~d}$, or frozen at $-10^{\circ} \mathrm{C}$ for later use, at which time they were brought to room temperature.

\section{Methods}

The methods used have been reported in the companion paper (Vlahopoulos and Jakinovich, 1986).

\section{Results}

\section{Discovery and characterization of PNP-Glu as an antagonist}

In order to synthesize chlorinated glycopyranosides that had been substituted at C-1, we used $p$-nitrophenyl $\alpha$-D-glucopyranoside (PNP-Glu) as our starting material. As is our practice whenever we obtain a new sugar derivative, we tested its effectiveness as a taste stimulant and noted that PNP-Glu at $0.03 \mathrm{M}$ (its maximum solubility) did not stimulate the gerbil's taste nerve (see Fig. 4). However, when we mixed it with $0.03 \mathrm{M}$ sucrose, we found that it specifically suppressed the sucrose response but had no effect on the sodium chloride, potassium chloride, or $\mathrm{HCl}$ responses (Fig. $1 A$ ). In addition, we noted that this inhibition was overcome by high concentrations of sucrose (Fig. 2), and that PNP-Glu ( $\mathrm{IC}_{50}=0.022 \mathrm{M}$ ) was a slightly more potent inhibitor than MAD-diCl-Gal $\left(\mathrm{IC}_{50}=0.048 \mathrm{M}\right)(\mathrm{Fig} .3)$.

\section{Structure-activity study}

To assess the inhibitory nature of PNP-Glu, we tested a number of aryl glycosides and some closely related aryl compounds, both alone (at 0.015 or $0.03 \mathrm{M}$ ) and in mixtures with $0.03 \mathrm{~m}$ sucrose (Table 1) and observed the following: 
A
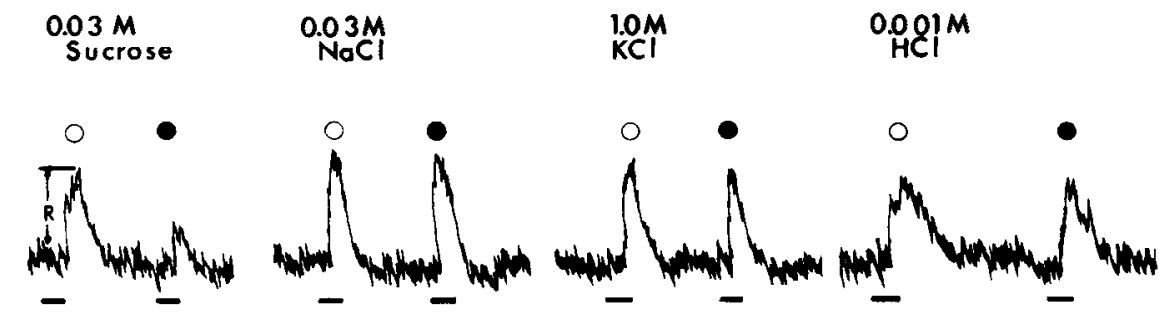

B
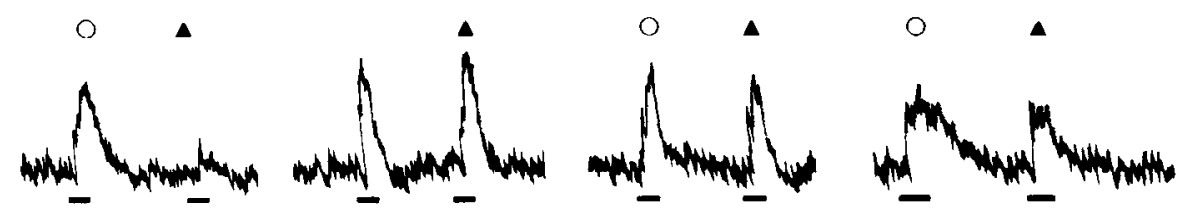

() $=$ St imulus alone

- Stimulus t0.03 M PNP - Glu

$30 \mathrm{sec}$

$\Delta=$ Stimulus $+0.015 \mathrm{M}$ Chloramphenicol

the measurement of the integrated responses.
1. Only compound I stimulated the gerbil's taste nerve to any significant degree $\left(>2 \frac{1}{2} \mathrm{SE}\right.$ ). The other compounds tested either stimulated very little, not at all, or sometimes caused a small depression of the summated nerve response, something we had observed with MAD-diCl-Gal (Vlahopoulos and Jakinovich, 1986).

2. In our previous study, none of the chlorinated methyl glycopyranosides tested proved to be a more potent inhibitor than MAD-diCl-Gal. However, in these experiments dealing with aryl derivatives, we have discovered 3 compounds (IX, $X$, and XI, Table 1) that are more potent inhibitors than PNP-Glu, our lead aryl compound. It is noteworthy that the stimulating potency of the methyl glycoside derivatives of compounds VIII, IX, and X (Jakinovich and Goldstein, 1976) is the reverse of the strength of antagonism of their aryl derivatives.

3. Among the readily available aryl glycosides tested, the position of the nitro group on the phenyl ring seems to play a role

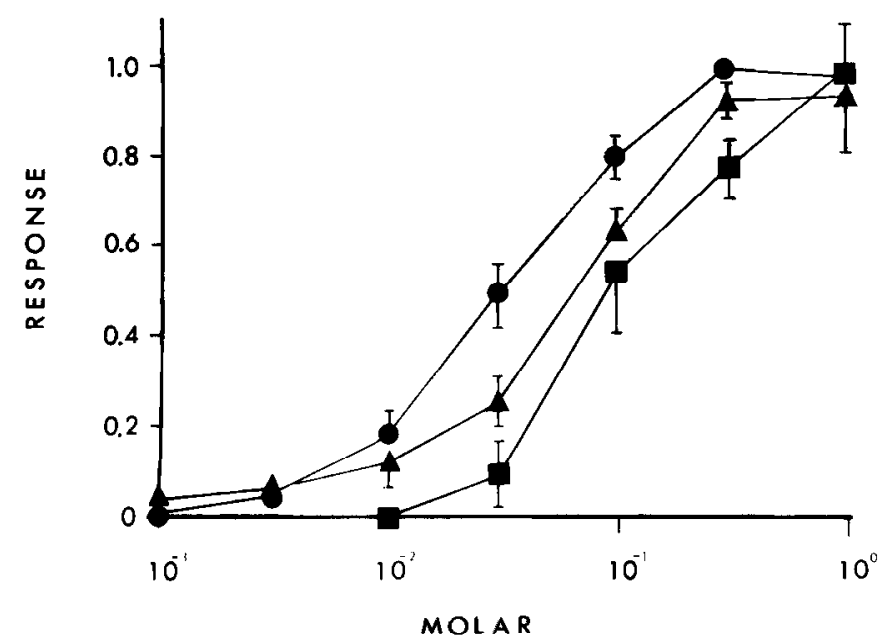

Figure 2. Effect of 0.03 м PNP-Glu ( $\square), n=4$, and 0.015 м CAP (A), $n=5$, on various concentrations of sucrose $(0), n=10, \pm 2$ SE (Vlahopoulos and Jakinovich, 1986). in the degree of inhibition. For example, the para derivative (compound IX) was a more potent inhibitor than the ortho derivative (compound VII) or the meta derivative (compound VI). Interestingly, the latter 2 compounds suppressed the sucrose response to the same degree.

4 . With regard to electronic properties, among the 3 major types of aryl glycosides tested ( $p$-amino, $p$-nitro, and phenylcompounds I, II, VIII), the $p$-nitro derivative (compound VIII) was the only one that inhibited.

5. As to the orientation of the substituent group at the $\mathrm{C}-1$ position, the alpha anomer of PNP-Glu (compound VIII) was a more potent inhibitor of the sucrose response than its beta anomer (compound $\mathrm{V}$ ).

6. Among the other compounds containing the $p$-nitrophenyl

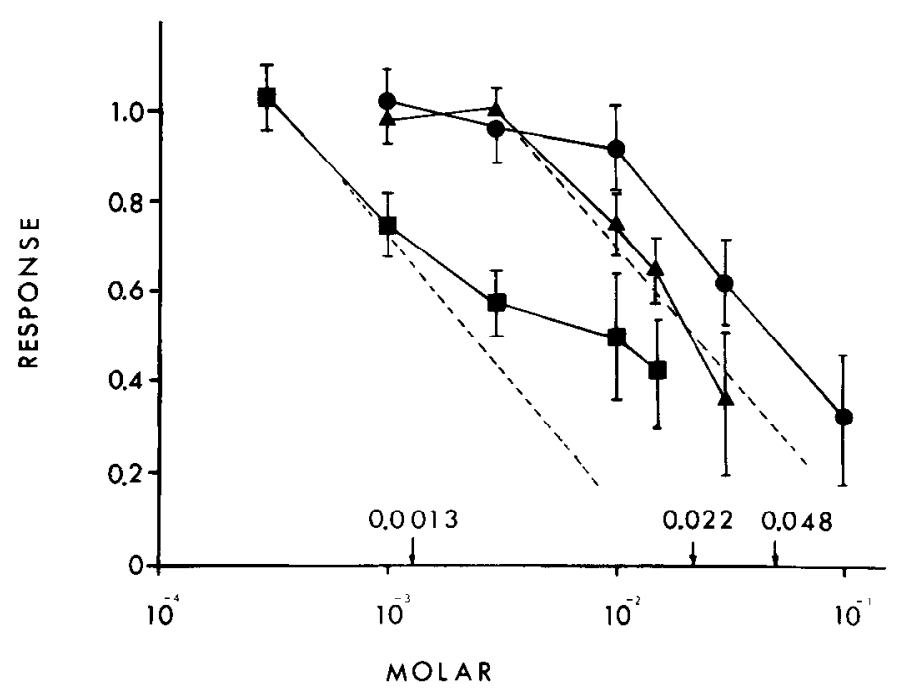

Figure 3. Effect of various concentrations of MAD-diCl-Gal (O), PNPGlu ( $\triangle$ ), and CAP $(\square)$ on the 0.03 m sucrose response. The dashed lines are parallel to the MAD-diCl-Gal response. The $\mathrm{IC}_{50}$ s (concentrations of the antagonist that inhibited the sucrose response by 50\%) are shown on the ahscissa. Bars, +2 SE. $n=9$ animals for MAD-diCl-Gal, 5-6 animals for PNP-Glu, and 6 animals for CAP. 
Table 1. Gerbil's chorda tympani nerve responses to some aryl glycopyranosides and related compounds and their effect in mixtures with $0.03 \mathrm{M}$ sucrose, ranked according to the response of the mixture

\begin{tabular}{|c|c|c|c|c|}
\hline Compound & $\begin{array}{l}\text { Response } \\
\pm 2 \mathrm{SE} \\
\end{array}$ & $\begin{array}{l}\text { Mixture } \\
\text { response } \\
\pm 2 \mathrm{SE} \\
\end{array}$ & $\begin{array}{l}\text { Change } \\
(\%)\end{array}$ & $n$ \\
\hline Sucrose (control) & 1.00 & 1.00 & & \\
\hline \multicolumn{5}{|l|}{$0.03 \mathrm{M}$ Concentration } \\
\hline I. $p$-Aminophenyl $\alpha$-D-glucopyranoside & $0.42 \pm 0.013$ & $1.36 \pm 0.11$ & 42 & 8 \\
\hline II. Phenyl $\alpha$-D-glucopyranoside & 0 & $1.10 \pm 0.10$ & 10 & 4 \\
\hline III. $p$-Nitrophenol & $0.13 \pm 0.14$ & $0.98 \pm 0.27$ & -2 & 4 \\
\hline IV. $p$-Nitrophenyl $\alpha$-glycerol & 0 & $0.92 \pm 0.12$ & -8 & 4 \\
\hline V. $p$-Nitrophenyl $\beta$-D-glucopyranoside & $0.07 \pm 0.08$ & $0.88 \pm 0.20$ & -12 & 4 \\
\hline VI. $n$-Nitrophenyl $\alpha$-D-galactopyranoside & $-0.09 \pm 0.11$ & $0.58 \pm 0.11$ & -42 & 4 \\
\hline VII. $O$-Nitrophenyl $\alpha$-D-galactopyranoside & $0.03 \pm 0.08$ & $0.58 \pm 0.22$ & -42 & 5 \\
\hline VIII. $p$-Nitrophenyl $\alpha$-D-glucopyranoside ${ }^{a}$ & 0 & $0.35 \pm 0.15$ & -65 & 5 \\
\hline IX. $p$-Nitrophenyl $\alpha$-D-galactopyranoside & 0 & $0.23 \pm 0.10$ & -77 & 7 \\
\hline \multicolumn{5}{|l|}{$0.015 \mathrm{M}^{6}$} \\
\hline VIII. $p$-Nitrophenyl $\alpha$-D-glucopyranoside & 0 & $0.70 \pm 0.19$ & -30 & 4 \\
\hline IX. $p$-Nitrophenyl $\alpha$-I)-galactopyranoside & 0 & $0.50 \pm 0.11$ & -50 & 4 \\
\hline X. $p$-Nitrophenyl $\alpha$-D-mannopyranoside & 0 & $0.47 \perp 0.15$ & -53 & 4 \\
\hline XI. Chloramphenicol & $0.05 \pm 0.06$ & $0.38 \pm 0.13$ & -62 & 6 \\
\hline
\end{tabular}

a PNP-Glu.

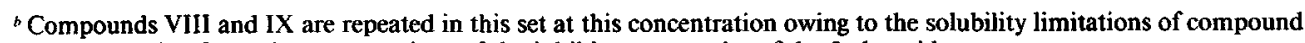
$X$, thus allowing for a direct comparison of the inhibitory potencies of the 3 glycosides.

cAP.

group that were tested as potential inhibitors, only one, CAP, was inhibitory.

\section{Characterization of CAP as an inhibitor}

In an attempt to assess the inhibitory specificity of CAP, we mixed it with a number of compounds and found that it had no effect on the sodium chloride, potassium chloride, or $\mathrm{HCl}$ responses (Fig. $1 B$ ). We also found that CAP's inhibition of the sucrose taste response was surmounted by high concentrations of sucrose (Fig. 2), and that it was a much better sucrose inhibitor $\left(\mathrm{IC}_{50}=0.0013 \mathrm{M}\right)$ than MAD-diCl-Gal $\left(\mathrm{IC}_{50}=0.048 \mathrm{M}\right)$ or PNPGlu $\left(\mathrm{IC}_{s 0}=0.022 \mathrm{M}\right.$ ) (see Fig. 3).

It should be noted that in the calculation of these inhibitory constants we noticed (see Fig. 3) that the inhibition of the sucrose response by CAP seems to be saturating at approximately the $40 \%$ level, on which we based our calculations. Furthermore, it appears from Figure 3 that the inhibition mechanism of CAP is quite different from that of PNP-Glu or MAD-diCl-Gal.

\section{The effect on the sucrose response by preexposure to}

\section{inhibitors}

Since it has been found that preexposing the human tongue to a solution of gymnemic acid for $2 \mathrm{~min}$, followed by rinsing, results in suppression of sweet taste for over an hour (Kurihara, 1969), we attempted to explore this phenomenon on the gerbil, using PNP-Glu and CAP. No effects of this nature were observed (Fig. 4). A description of the similar lack of effect using MADdiCl-Gal has already been published (Jakinovich, 1983).

\section{Optical rotations of mixtures of sucrose with} $P N P$-Glu and CAP

To assess the possibility that the above inhibitors were reacting with sucrose, thereby reducing its effective concentration, we compared the optical rotations of the various mixtures and found that the rotations were simply additive, indicating no interactions (Table 2).

\section{Discussion}

In the companion paper (Vlahopoulos and Jakinovich, 1986), we presented data indicating that methyl 4,6-dichloro-4,6dideoxy- $\alpha$-D-galactopyranoside (MAD-diCl-Gal) is a potentially useful inhibitor of the sucrose taste response in the gerbil. However, since MAD-diCl-Gal is poorly soluble, has a bitter taste, and is a weak inhibitor, its usefulness in behavioral experiments is limited. At that time, we also tried to determine whether MAD-diCl-Gal was a competitive, noncompetitive, or allosteric inhibitor and ruled out certain noncompetitive factors.

With regard to the present compounds of interest, $p$-nitrophenyl $\alpha$-D-glucopyranoside (PNP-Glu) and chloramphenicol (CAP), we have discovered that they are both more potent inhibitors than MAD-diCl-Gal, and have followed the same procedures in trying to determine the mechanism of their inhibition. Factors such as $\mathrm{pH}$ effects, compound interactions, bitterness, and anesthetic effects have been ruled out as contributing to the suppression of the sucrose response, on the basis of the following evidence:

1. $p H$ Effect. One possible explanation of the depressed response to the mixtures of PNP-Glu and sucrose, and CAP and sucrose is that the antagonists might be acting as acids, or con-

\begin{tabular}{|c|c|}
\hline $\begin{array}{l}\text { Solution } \\
(n=5)\end{array}$ & $\begin{array}{l}\text { Rotation } \\
{[\alpha]_{D}^{25}}\end{array}$ \\
\hline Sucrose, $0.03 \mathrm{~m}$ & +0.72 \\
\hline PNP-Glu, $0.03 \mathrm{M}$ & +1.94 \\
\hline CAP, $0.015 \mathrm{M}$ & -0.04 \\
\hline Mixtures of sucrose and PNP-Glu & +2.80 \\
\hline Mixture of sucrose and CAP & +0.80 \\
\hline
\end{tabular}


A
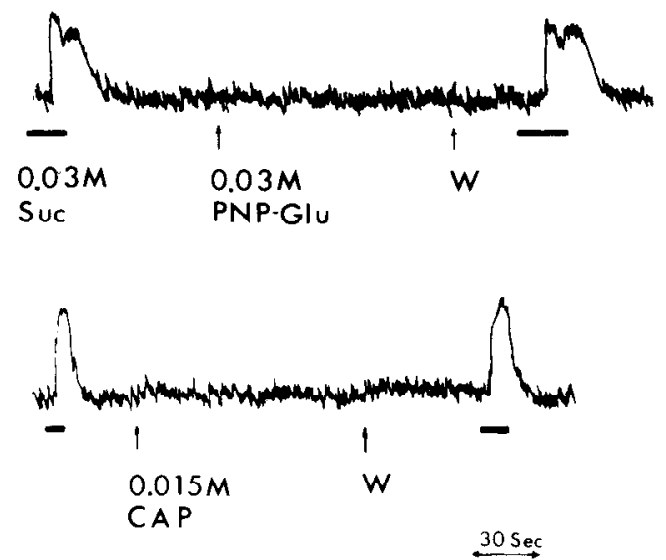

Figure 4. Effect of preexposure to PNP-Glu $(A)$ and CAP $(B)$ on the sucrose response. Bars indicate the addition of $0.03 \mathrm{M}$ sucrose to the flow system. The first arrows indicate the additions of $0.03 \mathrm{~m}$ PNP-Glu and $0.015 \mathrm{M}$ CAP. Arrow $W$ indicates the addition of the water rinse following that of the antagonist. The antagonist is added to the flow system for $2 \mathrm{~min}$, followed by a $30 \mathrm{sec}$ or $1 \mathrm{~min}$ water rinse.

tain an acidic impurity, since it is known that low $\mathrm{pH}$ depresses the sugar taste response in humans (Stone et al., 1969); gerbils (Jakinovich, 1982); hamsters (Hyman and Frank, 1980); and rats (Ogawa, 1969a, b). However, our $0.03 \mathrm{M}$ PNP-Glu and $0.015 \mathrm{M}$ CAP have pHs of 5.5 and 5.0 , respectively, which are more or less the same as the 5.6 of the air-equilibrated deionized water that has been stored in plastic carboys and in which they are dissolved. To check this further, we also conducted experiments using a pH buffer of 7.0 and found that these compounds still inhibited the sucrose response, all of which tends to rule out any $\mathrm{pH}$ effects.

2. Anesthetic effect. Our structure-activity study, together with the specificity of taste quality inhibition, i.e., the failure to suppress the $\mathrm{NaCl}$ or $\mathrm{HCl}$ response, as well as the failure of pretreatment to affect the sucrose response (Jakinovich, 1983) tend to rule out the possibility that PNP-Glu and CAP are behaving as nonspecific anesthetics, as reported by Von Skramlik (1963).

3. Compound interaction. To tcst the possibility that PNPGlu or CAP was reacting with sucrose, thus reducing its effective concentration, we examined the mixtures by polarimetry and found no evidence of reactions. However, it is still possible that the polarimeter may not be sufficiently sensitive to pick up small reactions.

4. Bitterness. One possible reason for PNP-Glu's or CAP's suppression of the sucrose response is their bitterness, since the behavioral suppression of a sweet taste by a bitter substance is well known (Bartoshuk, 1975). Based on our previous experiments (Vlahopoulos and Jakinovich, 1986), this is not the case.

5. Inhibition of protein synthesis by CAP. CAP is well known as an inhibitor of mitochondrial protein synthesis in eukaryotes (Schatz and Mason, 1974). However, since CAP's inhibitory effect in the gerbil is immediate and thus readily reversible, and since preexposure for at least $2 \mathrm{~min}$ has no effect on the gerbil's sucrose taste response, we rule this out as a possible mechanism.

On the other hand, we believe that the following pieces of evidence support the idea of specific receptor mechanisms and explain the inhibition of the gerbil's sucrose response by PNPGlu or CAP:

1. Surmountability. A crucial piece of evidence for competitive interaction (Ariens, 1964) is the fact that inhibition was surmounted in both cases by high concentrations of sucrose (Fig. 2).

2. Application methods. In addition, PNP-Glu and CAP must be mixed in solution with sucrose to be inhibitory. Moreover,
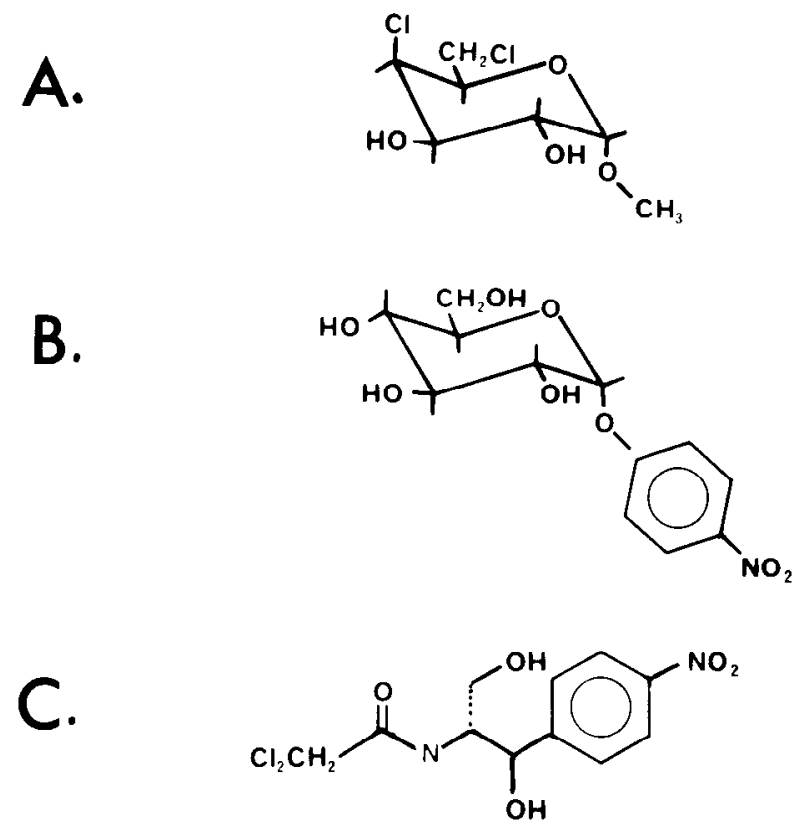

Figure 5. Structural comparison of $(A)$ MAD-diCl-Gal, (B) PNP-Glu, and $(C)$ CAP (Hahn and Gund, 1974).

a 2 min pretreatment of the gerbil's tongue with these compounds did not affect the responses, also lending credibility to the competitive mechanism.

3. Structure-activity. Of all the aryl glycopyranosides tested, the $\alpha$-glycosides of PNP-Glu proved to be much more potent than their $\beta$-counterparts (see Table 1). In the same vein, methyl $\alpha$-D-glucopyranoside and methyl $\alpha$-D-galactopyranoside were more potent stimuli than their $\beta$-counterparts (Jakinovich and Goldstein, 1976). This would also support the idea that, at least in the case of the glycosides of PNP-Glu, inhibitors and stimuli are binding at the same receptor site. In the case of CAP, we believe, from the data shown in Figures 2 and 3, that the mechanism may be different. However, at this point, we have insufficient structurc-activity data to make a definitive statement. It should also be pointed out that an allosteric receptor site cannot be ruled out in both cases.

Despite the seemingly diverse structures of these 3 types of compounds (methyl glycopyranosides, aryl glycopyranosides, and chloramphenicol), we feel that the same structure-activity rules exist for the first 2 groups of these inhibitors as were found to hold for the previously described sugar stimuli in the gerbil (see Jakinovich, 1985; Jakinovich and Goldstein, 1976). The inhibitory mechanism by CAP, however, seems to follow a slightly different path. With that in mind, we offer these present observations:

First of all, the most potent inhibitors within the MAD-diClGal derivatives and the PNP-Glu derivatives are the alpha sugars. This is what would be expected if these compounds were binding to the glucopyranosyl subsite of the sucrose receptor site (Jakinovich and Oakley, 1975), where there appears to be a steric preference for the alpha orientation for both excitation (Jakinovich and Goldstein, 1976) and inhibition (Vlahopoulos and Jakinovich, 1986).

With regard to $p$-nitrophenyl-glycopyranosides: We initially discovered that PNP-Glu was a slightly better inhibitor than MAD-diCl-Gal, and subsequent structure-activity studies showed that $p$-nitrophenyl $\alpha$-D-galactopyranoside and $p$-nitrophenyl $\alpha$-D-mannopyranoside were even slightly more potent, which again suggested that there is a balance between activation and inactivation within the receptor site. In this case, while the 
methyl glycosides, methyl $\alpha$-D-mannopyranoside, and methyl $\alpha$-D-galactopyranoside are poor stimulants (Jakinovich and Goldstein, 1976), their $p$-nitrophenyl derivatives are superior inhibitors. Assuming that these aryl glycopyranosides are binding within the sucrose site, we believe that the pyranosyl ring is binding to the glucopyranosyl subsite while the $p$-nitrophenyl ring is binding to the fructofuranosyl subsite, probably at the hydrophobic site that also binds the methyl group. However, there is also undoubtedly a definite electronic basis for this mechanism. For example, the $p$-amino phenyl derivative, as well as the phenyl derivative of PNP-Glu, does not inhibit. In fact, the $p$-amino phenyl $\alpha$-D-glucopyranoside is actually a good stimulant. On the other hand, phenyl $\alpha$-D-glucopyranoside does not stimulate at all.

In the case of CAP, we believe that its inhibitory mechanism is different from that of PNP-Glu and MAD-diCl-Gal. At first, when we discovered that CAP inhibited the gerbil's sucrose response, we hypothesized that since this compound was flexible (Fig. 5), possessed 2 chlorine atoms, hydroxyl groups, and a $p$-nitrophenyl group, and therefore had the ability to assume a structure similar to $p$-nitrophenyl diCl-Gal, a compound common to both PNP-Glu and MAD-diCl-Gal, its inhibition mechanism should be the same. However, Figure 3 clearly demonstrates that CAP's mechanism of inhibition is different, which leads to the conclusion that there exist at least 2 sucrose receptor sites, only 1 of which is antagonized by CAP.

\section{References}

Ariens, E. J. (1964) In Molecular Pharmacology, vol. 1, E. J. Aricns, ed., p. 363, Academic, New York.

Bartoshuk, L. M. (1975) Taste mixtures: Is mixture suppression related to compression? Physiol. Behav. 14: 643-649.

Hahn, F. E., and P. Gund (1974) A structural model of the chloramphenicol receptor site. In Topics in Infectious Diseases. Vol. I: Drug Receptor Interactions in Antimicrobial Chemotherapy, J. Drews and F. E. Hahn, eds., pp. 245-266, Springer, New York.
Hough, L., and S. P. Phadnis (1976) Enhancement in the sweetness of sucrose. Nature 263: 800 .

Hyman, A. M., and M. E. Frank (1980) Effects of binary taste stimuli in the neural activity of the hamster chorda tympani. J. Gen. Physiol. 76: 125-142.

Jakinovich, W., Jr. (1981) Stimulation of the gerbil's gustatory receptors by artificial sweeteners. Brain Res. 210:69-81.

Jakinovich, W., Jr. (1982) Stimulation of the gerbil's gustatory receptors by saccharin. J. Neurosci. 2: 49-56.

Jakinovich, W., Jr. (1983) Methyl 4,6-dichloro-4,6-dideoxy- $\alpha$-D-galactopyranoside: An inhibitor of sweet taste responses in gerbils. Science 219: 408-410.

Jakinovich, W., Jr. (1985) Stimulation of the gerbil's gustatory receptors by methyl glycopyranosides. Chem. Senses 10: 591-604.

Jakinovich, W., Jr., and I. J. Goldstein (1976) Stimulation of the gerbil's gustatory receptors by monosaccharides. Brain Res. 110:491504.

Jakinovich, W., Jr., and B. Oakley (1975) Comparative gustatory response in four species of gerbilline rodents. J. Comp. Physiol. 99: 89101.

Kurihara, Y. (1969) Antisweet activity of gymnemic acid $A_{1}$ and its derivatives. Life Sci. $8(1)$ : 537-543.

Ogawa, H. (1969a) Effects of metabolic inhibitors on taste nerve responses in the chorda tympani nerve of rats. Jpn. J. Physiol. 19:652662.

Ogawa, H. (1969b) Effects of $\mathrm{pH}$ on taste responses in the chorda tympani nerve of rats. Jpn. J. Physiol. 19: 670-681.

Schatz, G., and Mason, T. L. (1974) The biosynthesis of mitochondrial proteins. Annu. Rev. Biochem. 43: 51-87.

Stone, H., S. Oliver, and J. Kloehn (1969) Temperature and pH effects on the relative sweetness of suprathreshold mixtures of dextrose fructose. Percep. Psychophys. 5: 257-260.

Vlahopoulos, V., and W. Jakinovich, Jr. (1986) A structure-activity study on the sucrose taste antagonist methyl 4,6-dichloro-4,6-dideoxy- $\alpha$-D-galactopyranoside. J. Neurosci. 6: 2604-2610.

Von Skramlik, E. (1963) The fundamental substrates of taste. In Proceedings of the First International Symposium on Olfaction and Taste, Y. Zotterman, ed., pp. 125-132, Pergamon, Oxford. 